\title{
Comparative proteome analysis of the spinal dural arteriovenous fistula arterial draining vein with label-free quantitative proteomics
}

Peixi Liu

Fudan University Huashan Hospital Department of Neurosurgery

Yuan Shi

Fudan University Huashan Hospital Department of Neurosurgery

Sichen Li

Fudan University Huashan Hospital Department of Neurosurgery

Yingjun Liu

Fudan University Huashan Hospital Department of Neurosurgery

\section{Yingjie Zhou}

Huashan Hospital Fudan University Department of Hand Surgery

\section{Yaying Song}

Shanghai Jiao Tong University

Wei Zhu

Huashan Hospital

Qingzhu An ( $\sim$ anqingzhu@me.com )

\section{Research article}

Keywords: SDAVF draining vein, superficial temporal artery, superficial temporal vein, proteomics

Posted Date: January 1st, 2020

DOl: https://doi.org/10.21203/rs.2.19789/v1

License: (c) (i) This work is licensed under a Creative Commons Attribution 4.0 International License.

Read Full License 


\section{Abstract}

Background: Spinal dural arteriovenous fistula (SDAVF) is the most common spinal vascular shunt lesion. Although pathological changes in the SDAVF draining vein (SDAVF-DV) have been elucidated, protein changes remain enigmatic. We investigated protein changes in the SDAVF-DV.

Methods: Three SDAVF-DV samples were collected, and superficial temporal artery (STA) and superficial temporal vein (STV) samples were used as controls. After quantification and enzymolysis of the proteins, label-free quantitative proteomics was performed, and the peptide mixture was fractionated and analysed by liquid chromatography tandem mass spectrometry (LC-MS/MS) to identify the differentially expressed proteins. Bioinformatics analysis of the differentially expressed proteins was also performed using Gene Ontology (GO), Kyoto Encyclopedia of Genes and Genomes (KEGG) and protein-protein interaction (PPI) network analyses.

Results: Compared with the STA, the SDAVF-DV had 195 upregulated proteins and 303 downregulated proteins. GO analysis showed that the most differential GO terms in each category were the adenylate cyclase-modulating $\mathrm{G}$ protein-coupled receptor signalling pathway, U6 snRNP and SH3 domain binding. KEGG pathway analysis showed that the most differentially expressed protein pathway was focal adhesion. Compared with the STV, the SDAVF-DV had 158 upregulated proteins and 362 downregulated proteins. $\mathrm{GO}$ analysis showed that the most differential $\mathrm{GO}$ terms in each category were lamellipodium assembly, U6 snRNP, and SH3 domain binding. KEGG pathway analysis showed that the most differentially expressed protein pathway was dilated cardiomyopathy. The PPI analysis revealed PPIs among the top 300 proteins.

Conclusions: We demonstrated that the SDAVF-DV showed specific protein expression changes under long-period venous hypertension. The results of the present study will provide insights into the pathogenesis of SDAVF formation at the protein level. The proteomic results provide a scientific foundation for further study to explore the pathophysiological mechanism of SDAVF.

\section{Background}

Spinal dural arteriovenous fistula (SDAVF) is the most common spinal vascular shunt lesion characterized by an abnormal connection between a radicular meningeal artery and a radicular medullary vein. As venous connections drain to radicular veins, the draining vein shows gradual arterialization. Because of venous hypertension, clinical presentations and progressive myelopathy can be assessed.

In SDAVF, venous drainage is provided by longitudinal spinal veins linked together and to the epidural network $^{1,2}$. In many clinical case reports, the arterialized SDAVF draining vein (SDAVF-DV) was identified easily after opening the dura during an operation. The pathology of the arterialized SDAVF-DV was mentioned in a previous study. However, the protein changes in this arterialized vein under high intravascular pressure remain enigmatic. 
In the present study, we used quantitative proteomics to compare the SDAVF-DV with the superficial temporal artery (STA) and superficial temporal vein (STV) to show different protein expression levels under venous hypertension. The results of our present study might provide insights into the pathogenesis of SDAVF formation at the protein level.

\section{Methods}

\section{Ethics statement}

The current study was examined and approved by the Ethics Committee of Huashan Hospital, Fudan University. Each participant provided their written informed consent to participate in this study.

\section{Patients and tissue sample preparation}

Three SDAVF-DVs were removed after microsurgery ligation. Three STAs and three STVs were obtained from patients with intracranial tumours via the extended pterional approach ${ }^{3,4}$. We used the samples from each group for the comparative proteomics analysis. The tissues used for the proteomics analysis were immediately frozen in liquid nitrogen and stored at $-80^{\circ} \mathrm{C}$. The SDAVF-DVs, STAs and STVs were homogenized in a $4 \%$ SDS, $100 \mathrm{mM}$ Tris- $\mathrm{HCl}$ and $100 \mathrm{mM}$ DTT solution. Then, a fluorescence assay was conducted to determine the total protein concentration. Approximately $200 \mu \mathrm{g}$ of total protein from the tissues was proteolysed on a 10-kDa filter (PALL Life Sciences, Shanghai, China) using a Filter Aided Sample Preparation (FASP) protocol as described in detail elsewhere ${ }^{5}$. The peptide solution was transferred to a Solid Phase Extraction Cartridge (Empore $7 \mathrm{~mm} / 3 \mathrm{~mL}$ ) for desalting and clean-up. The peptide samples were resuspended in water with $0.1 \%$ formic acid $(\mathrm{v} / \mathrm{v})$, and the protein content was estimated by UV light spectral density at $280 \mathrm{~nm}^{6}$ prior to analysis by nano-liquid chromatography tandem mass spectrometry (N-LC-MS/MS).

\section{Label-free quantitative analysis and data processing}

Trypsin-digested peptides from the tissues were analysed by LC-MS/MS; each sample was analysed twice. All raw Xcalibur files acquired from the MS runs were analysed using the default settings of MaxQuant software (version 1.3.0.5) with minor modifications as previously described ${ }^{7}$. Hierarchical clustering was performed with MEV software (v4.6, TIGR). The differentially expressed proteins $(p<0.05)$ were analysed by hierarchical clustering to identify potential markers capable of classifying all samples.

The clustering pattern and expression analyses and volcano plots were based on $\mathrm{R}$ software according to standardized data. Venn diagrams of the characteristics of each of the three groups of differentially expressed proteins were generated.

The Gene Ontology (GO) and enrichment analyses of the dysregulated proteins in this experiment were based on the publicly available databases DAVID 6.7 (http://david.abcc.ncifcrf.gov/) and QuickGO (http://www.ebi.ac.uk/QuickGO/). 
The genomic, chemical and systemic functions of the dysregulated proteins were analysed and enriched by Kyoto Encyclopedia of Genes and Genomes (KEGG) analysis (http://www.kegg.jp/kegg/pathway.html). The significance of differential protein enrichment in each pathway entry was calculated using the hypergeometric distribution test and is expressed as the $p$ value. Predicted protein-protein interaction (PPI) networks for these differentially expressed proteins were constructed using the STRING database (http://string.embl.de/) and Cytoscape software (http://www.cytoscape.org/).

\section{Statistical analysis}

The statistical analysis was performed with IBM SPSS, and the graphs were generated with GraphPad Prism software. The significance of differences between two groups in the proteomics analysis was assessed using one-way analysis of variance (ANOVA). Proteins were defined as significantly differentially expressed when the ratio was ${ }^{3} 2$ or $£ 0.5$ in the SDAVF-DV compared with normal tissues ( $p$ $<0.01)$.

\section{Results}

\section{Identification of differentially expressed proteins in the SDAVF-DVs, STAs and STVs}

Three paired SDAVF-DV, STA and STV tissue samples were analysed in the initial discovery phase.

An equal amount of protein from each tissue was digested. Then, the peptides were analysed by N-LCMS/MS. Using MaxQuant (version 1.3.0.5), we identified 2829 non-redundant proteins with a local false discovery rate (FDR) $<1 \%$ and at least two unique peptides per protein. The label-free quantification (LFQ) intensity ratios for the 2829 proteins were calculated, and significant differences in the protein expression levels between two tissues were determined using a t-test $(p<0.05)$. Compared with the STA, the SDAVFDV had 195 significantly upregulated proteins and 303 significantly downregulated proteins. Compared with the STV, among the 520 proteins that exhibited significant differences, 158 were significantly upregulated and 362 were significantly downregulated in the SDAVF-DV (Figure 1A). When the three groups were combined, 480 differentially expressed proteins were identified (shown in the heatmap in Figure 1B). Venn analysis showed the variation and commonalities of different proteins in each group. A total of 1026 proteins were expressed in all groups, and 150 proteins were identified only in the SDAVF-DV (Figure 1C).

\section{GO analysis}


We performed GO analysis to analyse the differentially expressed proteins. When comparing the SDAVFDV with the STA, most of differential GO terms expressed in each category were the adenylate cyclasemodulating $\mathrm{G}$ protein-coupled receptor signalling pathway, U6 snRNP and $\mathrm{SH} 3$ domain binding. We examined the top ten up- and downregulated $\mathrm{GO}$ terms in the biological processes, cellular components and molecular functions categories with 2.0-fold $(p<0.05)$ differential gene expression (Table 1$)$.

When comparing the SDAVF-DV with the STV, and the most differential GO terms expressed in each category were lamellipodium assembly, U6 snRNP, and SH3 domain binding.

The top ten up- and downregulated GO terms based on the comparison of the SDAVF-DV and STV are also listed in Table 2. In GO classification, 93 differentially enriched GO terms were found between the SDAVF-DV and STA: $60 \mathrm{GO}$ terms were upregulated, and 33 were downregulated (Figure 2A). Compared with the STV, the SDAVF-DV had 109 differentially enriched GO terms: 31 terms were upregulated, and 78 were downregulated (Figure 2B).

\section{KEGG pathway analysis}

The KEGG pathway analysis of these differentially expressed proteins also demonstrated related pathways. Figure 3 shows the number of proteins in each KEGG pathway and the $p$ value of the top 20 pathways. Compared with the STA, the top three differentially expressed protein pathways were focal adhesion, the PI3K-Akt signalling pathway and the extracellular matrix (ECM)-receptor interaction. Compared with the STV, the top three differentially expressed pathways were dilated cardiomyopathy, hypertrophic cardiomyopathy and adrenergic signalling in cardiomyocytes.

\section{PPI analysis}

We used the STRING database to analyse the differentially expressed proteins, obtain the interactions/relationships among the differentially expressed proteins and calculate the combined score. We selected the top 300 proteins and found significant PPIs among them (Figures $\mathbf{5}$ and $\mathbf{6}$ ). Compared with the STA and STV, the SDAVF-DV showed up- and downregulated proteins, and the top three interaction proteins are listed in Table 3.

\section{Discussion}

SDAVF is a common arteriovenous shunt located inside the dura mater close to the spinal nerve root ${ }^{8}$. Venous hypertension, which induces medullar venous outflow disturbances, results in chronic hypoxia and congestive myelopathy ${ }^{9}$. The direct intraoperative measurement of the vascular pressure in the fistula can be as high as $74 \%$ of the systemic arterial pressure ${ }^{10,11}$. This finding may explain why, in 
some patients, symptoms become worse during physical activity with a concomitant increase in arterial pressure $^{12,13}$. Under long-period venous hypertension, draining vein arterialization begins.

A clear understanding of the mechanism of SDAVF development is still lacking. Our study was the first to perform a comparative proteome analysis and show the differential expression of proteins in arterialized SDAVF-DVs compared with normal arteries and veins. In general, most of the proteins were the same between the three groups. Because of its special pathophysiology, the SDAVF-DV showed specific protein expression compared with the STA and STV.

In the intraoperative observation, the SDAVF-DV showed arterial morphology. A. Thron proposed a hypothesis based on spine arteriovenous shunt anatomy ${ }^{9}$. Keisuke Takai showed that the vessel wall of the proximal subarachnoid portion of the intradural draining vessels was irregularly thickened by collagen and exhibited elastic fibrosis and was without a continuous internal elastic lamina and a regular smooth muscle layer. The diameter of the vessels was significantly enlarged ${ }^{14}$.

After GO analysis, the SDAVF-DV showed a decrease in smooth muscle contractile fibres, which might indicate smooth muscle cell dysfunction. This might be induced by long-range venous hypertension stretching on the SDAVF-DV. Stretch plays an important role in maintaining smooth muscle cell function and regulating inflammation. A former study showed that mechanical stretch-induced endoplasmic reticulum stress, apoptosis and inflammation contribute to thoracic aortic aneurysm and dissection ${ }^{15}$. In our research, we also identified that mechanical stretching could induce smooth muscle cell changes from a contract phenotype to an inflammatory phenotype ${ }^{16,17}$. The regulation of inflammatory factors is related to the hypothesis on SDAVF formation. In THE KEGG and PPI analyses, the ECM and focal adhesion showed obvious changes. Degeneration of the ECM is primarily induced by the secretion of inflammatory cytokines and cell infiltration in cerebral vascular disease ${ }^{18,19}$. During SDAVF formation, inner vessel wall inflammation might contribute to an insufficient ECM and trigger changes in pathological proteins.

\section{Conclusions}

To our knowledge, few studies have focused on the SDAVF-DV. However, several researchers have investigated its pathological characteristics ${ }^{14,20}$. We first examined protein changes to determine whether the lesion vessel was an artery, vein or vein-to-artery transition.

Most previous studies have revealed the histology and anatomy of the SDAVF-DV. Based on intraoperative findings, we demonstrated protein changes in the arterial SDAVF-DV. Our study adds new information on the formation of SDAVF to the realm of protein changes in the draining vein using proteomics. This finding may shed light on the mechanism of SDAVF formation.

\section{Abbreviations}


SDAVF= Spinal dural arteriovenous fistula

SDAVF-DV= Spinal dural arteriovenous fistula draining vein

STA = superficial temporal artery

STV= superficial temporal vein

KEGG= Kyoto Encyclopedia of Genes and Genomes (http://www.genome.jp/kegg/)

\section{Declarations}

\section{Availability of data and materials:}

The datasets used in the current study are available from the corresponding author on reasonable request.

\section{Declaration:}

- Ethics approval and consent to participate: The study was reviewed and approved by the Ethics Committee of Huashan Hospital, Fudan University. Each participant provided their written informed consent to participate in this study.

- Consent for publication: Not applicable.

- Availability of data and materials: All the materials and data are freely available.

- Competing interests: The authors declare that they have no competing interests.

- Funding $₫ T$ The Outstanding Academic Leaders Program of Shanghai Municipal Commission of Health and Family Planning (No. 2017BR006 to WZ) $₫$ National Natural Science Foundation of China (No. 81571102, No. 81870911 to WZ; No. 81801148 to PL)

- Authors' contributions $₫ \mathrm{PL}$ : conception and design of study, provision of study materials, collection and/or assembly of data, data analysis and interpretation, manuscript writing, and final approval of manuscript. YS: data analysis and interpretation, manuscript writing. SL: database input and data interpretation. YL: database input and data interpretation. YYS: manuscript writing. YZ: Data analysis. WZ: provision of study and revision and final approval of manuscript. QA: conception and design of study and revision and final approval of manuscript. PL and YS contributed to this work equally. WZ and QA are co-corresponding authors of this article.

- Acknowledgement囚Not applicable.

\section{References}


1. Amanieu C, Hermier M, Peyron N, Chabrol A, Deiana G, Manera L. Spinal dural arteriovenous fistula. Diagn Interv Imaging. 2014;95:897-902

2. Krings T, Geibprasert S. Spinal dural arteriovenous fistulas. AJNR Am J Neuroradiol. 2009;30:639-648

3. Hasan D, Hashimoto T, Kung D, Macdonald RL, Winn HR, Heistad D. Upregulation of cyclooxygenase2 (COX-2) and microsomal prostaglandin E2 synthase-1 (mPGES-1) in wall of ruptured human cerebral aneurysms: Preliminary results. Stroke. 2012;43:1964-1967

4. Wang J, Yu L, Huang $X$, Wang Y, Zhao J. Comparative proteome analysis of saccular intracranial aneurysms with iTRAQ quantitative proteomics. J Proteomics. 2016;130:120-128

5. Neilson KA, Ali NA, Muralidharan S, Mirzaei M, Mariani M, Assadourian G, Lee A, van Sluyter SC, Haynes PA. Less label, more free: Approaches in label-free quantitative mass spectrometry. Proteomics. 2011;11:535-553

6. Zhuang Y, Ma F, Li-Ling J, Xu X, Li Y. Comparative analysis of amino acid usage and protein length distribution between alternatively and non-alternatively spliced genes across six eukaryotic genomes. Mol Biol Evol. 2003;20:1978-1985

7. Sun X, Zhang H, Luo L, Zhong K, Ma Y, Fan L, Fu D, Wan L. Comparative proteomic profiling identifies potential prognostic factors for human clear cell renal cell carcinoma. Oncol Rep. 2016;36:3131-3138

8. Kendall BE, Logue V. Spinal epidural angiomatous malformations draining into intrathecal veins. Neuroradiology. 1977;13:181-189

9. Thron A, Krings T, Otto J, Mull M, Schroeder JM. The transdural course of radicular spinal cord Veins-A microangiographical and microscopical study. Clin Neuroradiol. 2015;25:361-369

10. Hassler W, Thron A. Flow velocity and pressure measurements in spinal dural arteriovenous fistulas. Neurosurg Rev. 1994;17:29-36

11. Hassler W, Thron A, Grote EH. Hemodynamics of spinal dural arteriovenous fistulas. An intraoperative study. J Neurosurg. 1989;70:360-370

12. Aminoff MJ, Barnard RO, Logue V. The pathophysiology of spinal vascular malformations. J Neurol Sci. 1974;23:255-263

13. Khurana VG, Perez-Terzic CM, Petersen RC, Krauss WE. Singing paraplegia: A distinctive manifestation of a spinal dural arteriovenous fistula. Neurology. 2002;58:1279-1281

14. Takai K, Komori T, Taniguchi M. Microvascular anatomy of spinal dural arteriovenous fistulas: Arteriovenous connections and their relationships with the dura mater. J Neurosurg Spine. 2015;23:526-533

15. Jia LX, Zhang WM, Zhang HJ, Li TT, Wang YL, Qin YW, Gu H, Du J. Mechanical stretch-induced endoplasmic reticulum stress, apoptosis and inflammation contribute to thoracic aortic aneurysm and dissection. J Pathol. 2015;236:373-383

16. Liu P, Song Y, Zhou Y, Liu Y, Qiu T, An Q, Song J, Li P, Shi Y, Li S, Quan K, Yang GY, Zhu W. Cyclic mechanical stretch induced smooth muscle cell changes in cerebral aneurysm progress by reducing collagen type IV and collagen type VI levels. Cell Physiol Biochem. 2018;45:1051-1060 
17. Liu P, Shi Y, Fan Z, Zhou Y, Song Y, Liu Y, Yu G, An Q, Zhu W. Inflammatory smooth muscle cells induce endothelial cell alterations to influence cerebral aneurysm progression via regulation of integrin and VEGF expression. Cell Transplant. 2019;28:713-722

18. Chalouhi N, Ali MS, Jabbour PM, Tjoumakaris SI, Gonzalez LF, Rosenwasser RH, Koch WJ, Dumont AS. Biology of intracranial aneurysms: Role of inflammation. J Cereb Blood Flow Metab. 2012;32:1659-1676

19. Starke RM, Raper DM, Ding D, Chalouhi N, Owens GK, Hasan DM, Medel R, Dumont AS. Tumor necrosis factor-alpha modulates cerebral aneurysm formation and rupture. Trans/ Stroke Res. 2014;5:269-277

20. Benhaiem N, Poirier J, Hurth M. Arteriovenous fistulae of the meninges draining into the spinal veins. A histological study of 28 cases. Acta Neuropathol. 1983;62:103-111

\section{Tables}

Table 1 Top 10 GO term (SDAVF-DV vs STA) 


\begin{tabular}{|c|c|c|}
\hline Category & Top 10 upregulated GO term & Top 10 downregulated GO term \\
\hline \multicolumn{3}{|c|}{ Biological process } \\
\hline & $\begin{array}{l}\text { adenylate cyclase-modulating G-protein coupled } \\
\text { receptor signaling pathway }\end{array}$ & translation reinitiation \\
\hline & lamellipodium assembly & IRES-dependent translational initiation \\
\hline & lamellipodium morphogenesis & ribosome disassembly \\
\hline & regulation of epithelial cell migration & regulation of growth \\
\hline & regulation of microtubule-based process & regulation of cardiac muscle hypertrophy \\
\hline & wound healing & $\begin{array}{l}\text { positive regulation of calcineurin-NFAT } \\
\text { signaling cascade }\end{array}$ \\
\hline & regulation of receptor recycling & plasma membrane repair \\
\hline & ESCRT III complex disassembly & $\begin{array}{l}\text { regulation of vascular endothelial growth factor } \\
\text { receptor signaling pathway }\end{array}$ \\
\hline & regulation of osteoclast differentiation & prostaglandin biosynthetic process \\
\hline & trophectodermal cell differentiation & secretion \\
\hline \multicolumn{3}{|c|}{ Cellular component } \\
\hline & nuclear pore outer ring & U6 snRNP \\
\hline & GATOR2 complex & Lsm1-7-Pat1 complex \\
\hline & mitochondrial envelope & ESCRT III complex \\
\hline & elastic fiber & cytoplasmic side of plasma membrane \\
\hline & GAIT complex & membrane coat \\
\hline & intermediate filament & endoplasmic reticulum exit site \\
\hline & neurofilament cytoskeleton & nuclear outer membrane \\
\hline & microfibril & rough endoplasmic reticulum \\
\hline & membrane raft & LINC complex \\
\hline & late endosome membrane & smooth muscle contractile fiber \\
\hline \multicolumn{3}{|c|}{ Molecular function } \\
\hline & SH3 domain binding & phospholipid binding \\
\hline & antioxidant activity & protein disulfide oxidoreductase activity \\
\hline & $\begin{array}{l}\text { ATP-dependent } \mathrm{NAD}(\mathrm{P}) \mathrm{H} \text {-hydrate dehydratase } \\
\text { activity }\end{array}$ & lyase activity \\
\hline & $\begin{array}{l}\text { ADP-dependent NAD(P)H-hydrate dehydratase } \\
\text { activity }\end{array}$ & glutathione binding \\
\hline & phosphatidylinositol phospholipase $\mathrm{C}$ activity & prostaglandin-E synthase activity \\
\hline & phospholipase $\mathrm{C}$ activity & catalytic activity \\
\hline & metallopeptidase activity & $\begin{array}{l}\text { protein serine/threonine phosphatase inhibitor } \\
\text { activity }\end{array}$ \\
\hline & G-protein coupled serotonin receptor binding & peroxidase activity \\
\hline & 3-hydroxyacyl-CoA dehydrogenase activity & interleukin-1 receptor antagonist activity \\
\hline & acetyl-CoA C-acyltransferase activity & proteinase activated receptor binding \\
\hline
\end{tabular}

Table 2 Top 10 GO term (SDAVF-DV vs STV) 


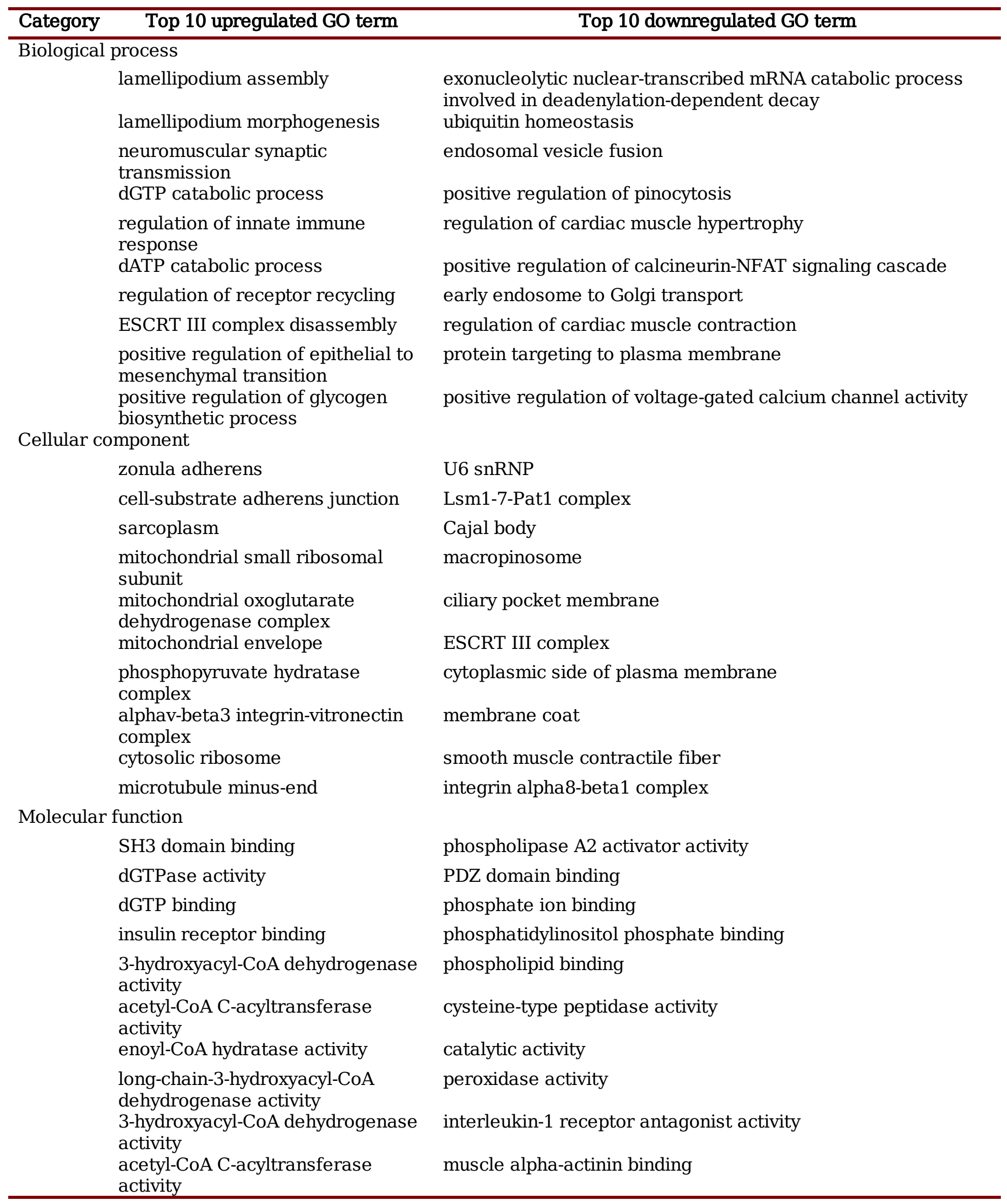

Table 3 Top 3 interaction protein number proteins 


\begin{tabular}{|c|c|c|}
\hline & Gene ID & Upregulated protein \\
\hline \multicolumn{3}{|c|}{ SDAVF-DV vs STA } \\
\hline & P63261 & Actin, cytoplasmic 2 \\
\hline \multirow[t]{3}{*}{ Upregulated } & P07814 & Bifunctional glutamate/proline--tRNA ligase \\
\hline & P62750 & 60S ribosomal protein L23a \\
\hline & P02751 & Fibronectin \\
\hline \multirow[t]{2}{*}{ Downregulated } & Q15149 & Plectin \\
\hline & P02452 & Collagen alpha-1(I) chain \\
\hline \multicolumn{3}{|c|}{ SDAVF-DV vs STV } \\
\hline & P00738 & Haptoglobin \\
\hline \multirow[t]{3}{*}{ Upregulated } & P02652 & Apolipoprotein A-II \\
\hline & P02765 & Alpha-2-HS-glycoprotein \\
\hline & P19652 & Alpha-1-acid glycoprotein 2 \\
\hline \multirow[t]{2}{*}{ Downregulated } & P10909 & Clusterin \\
\hline & P14780 & Matrix metalloproteinase-9 \\
\hline
\end{tabular}

\section{Figures}


A

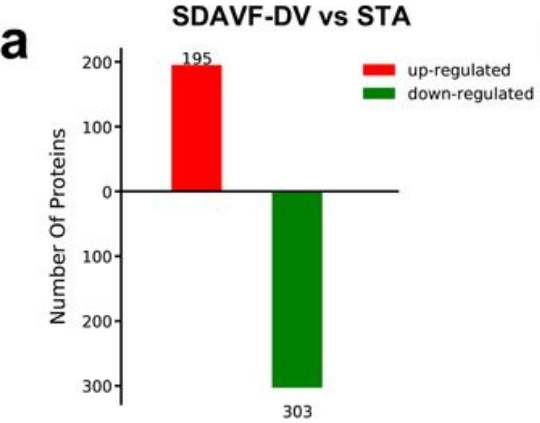

c

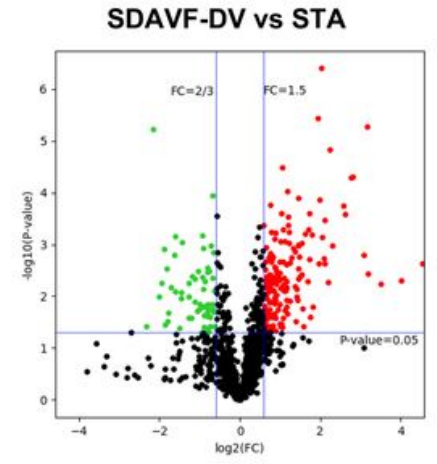

b

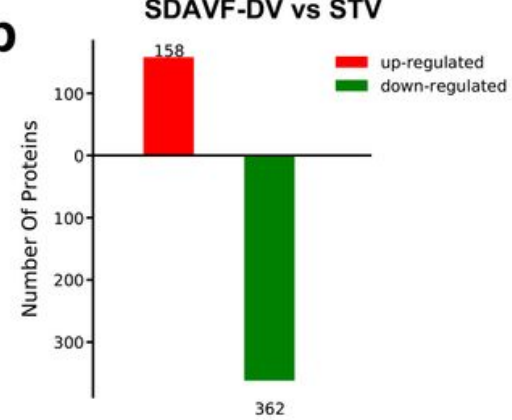

d

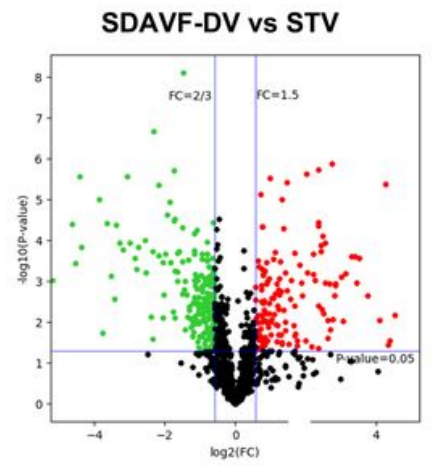

B

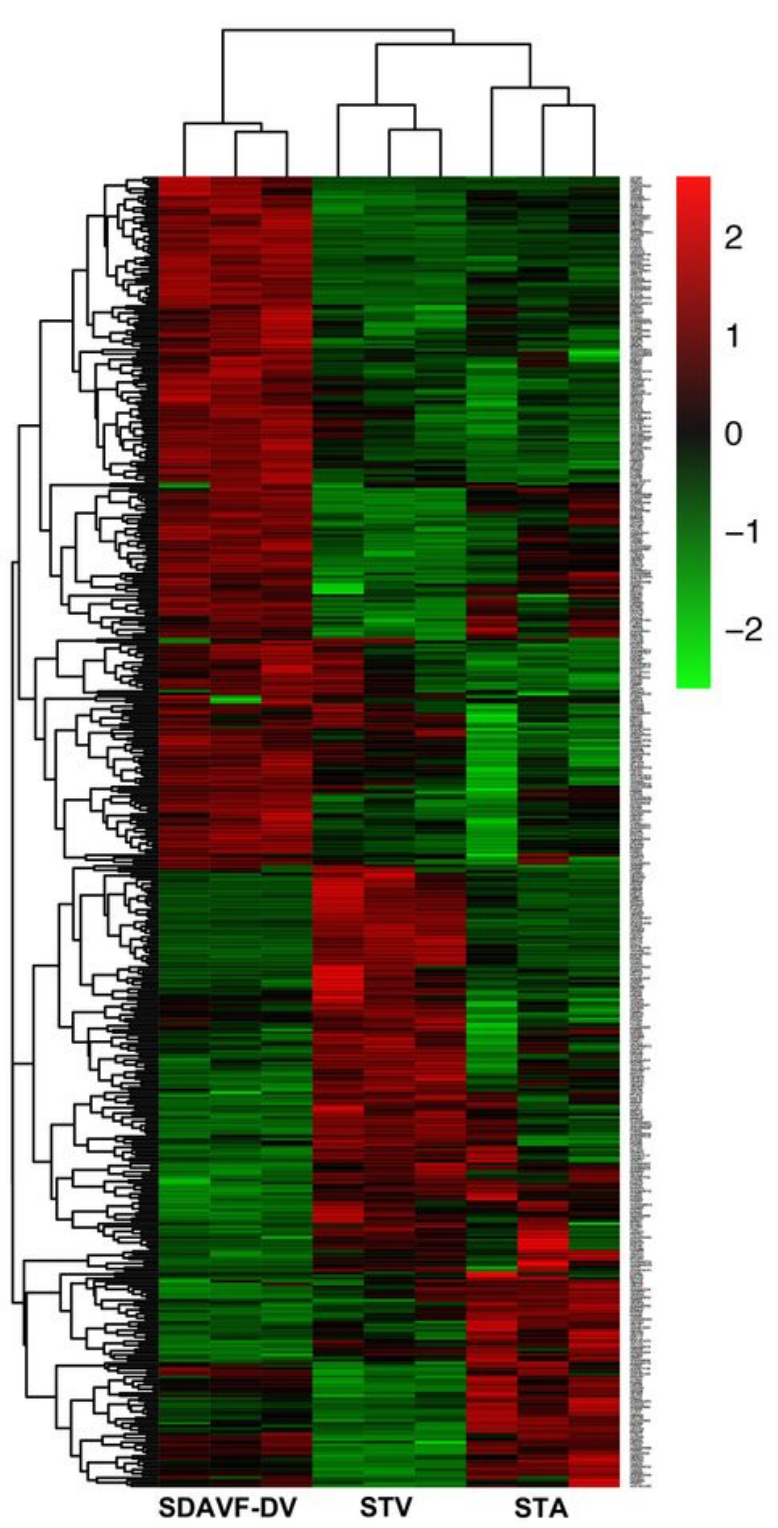

Figure 1

Differentially expressed proteins identified by label-free quantitative proteomics. A. Significant differences in protein levels in the SDAVF-DV compared with the STA (a) and STV (b). Volcano plot showing protein changes in the SDAVF-DV compared with the STA (c) and STV (d). B. Heatmap of 480 proteins expressed in the SDAVF-DV, STA and STV. C. Venn diagram showing overlap of protein expression. 
A Gene Ontology Classification (SDAVF-DV vs STA)

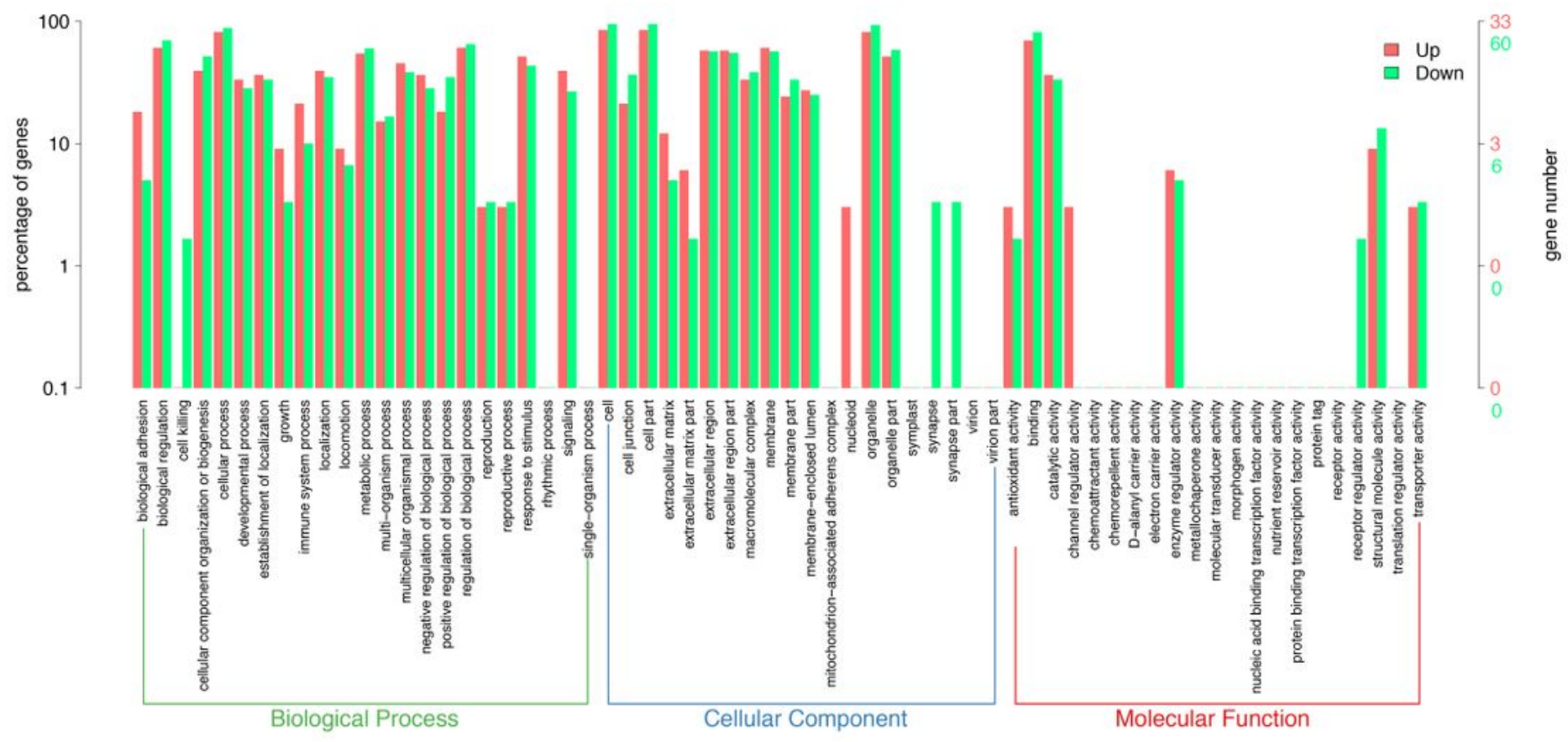

B

\section{Gene Ontology Classification (SDAVF-DV vs STV)}

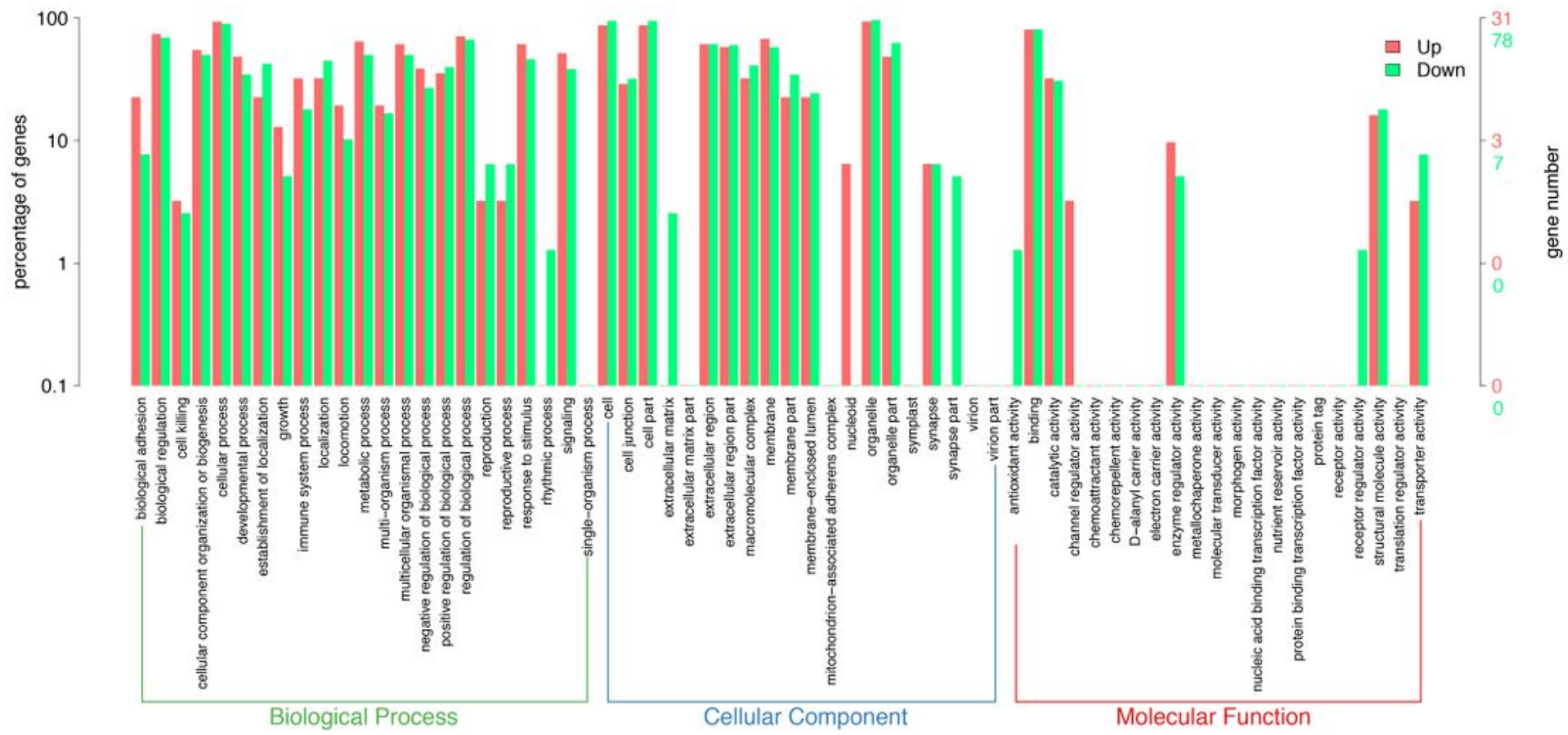

Figure 2

GO analysis of significantly dysregulated proteins and changes in the SDAVF-DV. GO analysis of the upand downregulated GO terms for the 2.0-fold differentially expressed genes associated with the biological process, cellular component and molecular function categories when comparing the SDAVF-DV with the STA (A) and STV (B). 

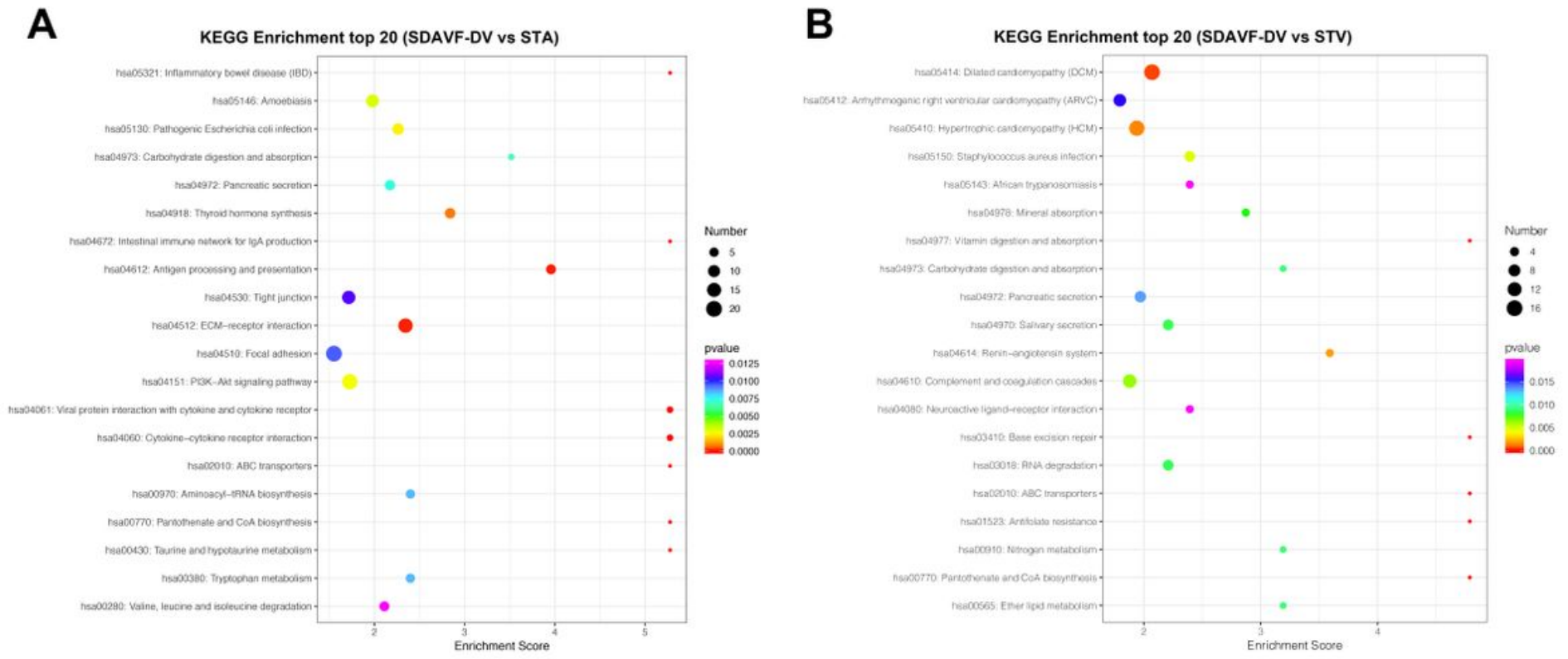

\section{Figure 3}

KEGG pathway analysis. The bubble chart shows the KEGG pathway analysis of the top 20 differentially expressed genes when comparing the SDAVF-DV with the STA (A) and STV (B). 


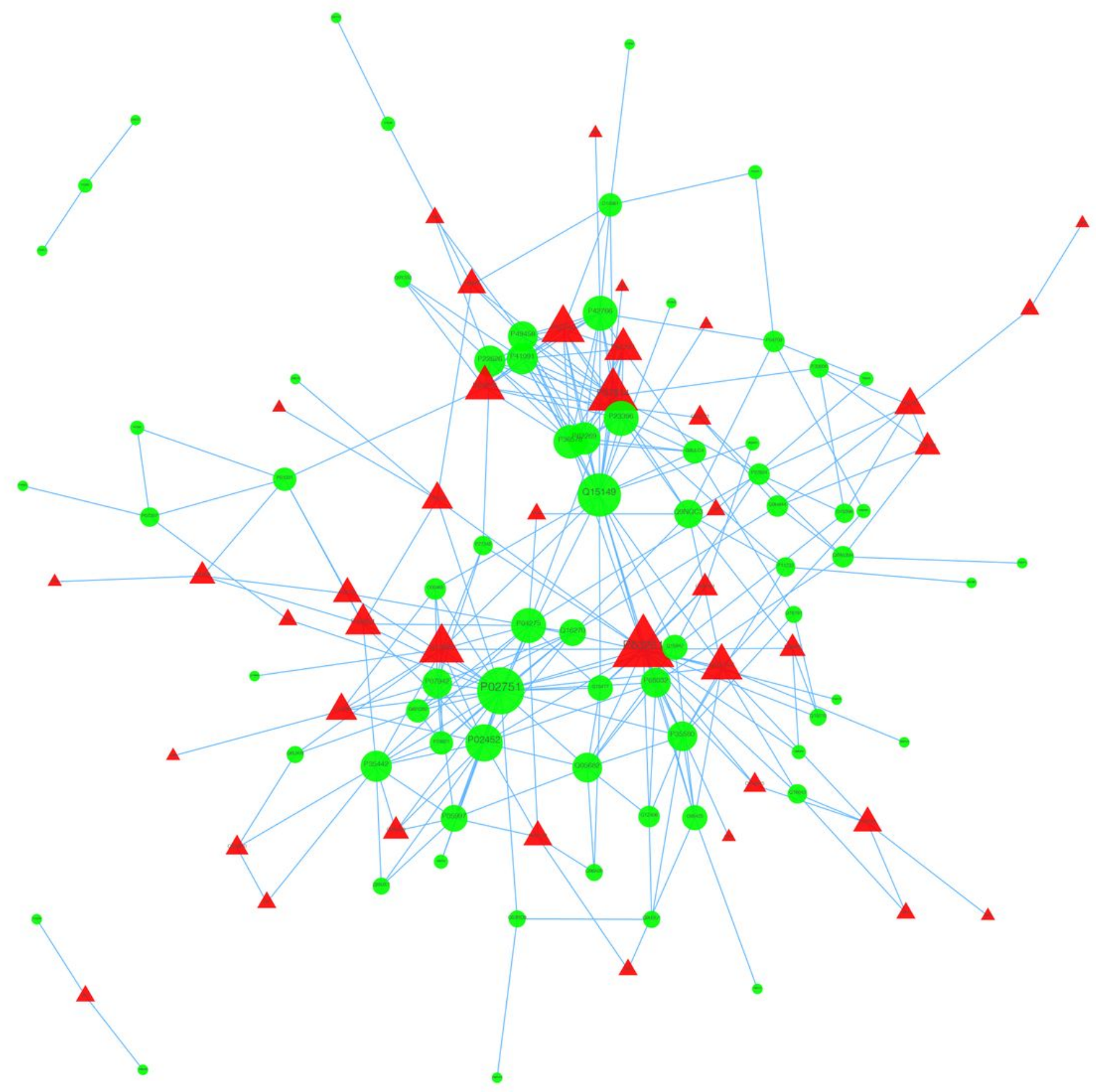

Figure 4

Visualization of PPIs between the SDAVF-DV and STA. Visualization of PPIs for the top 300 proteins using STRING analysis. Red represents upregulated proteins, and green represents downregulated proteins. 


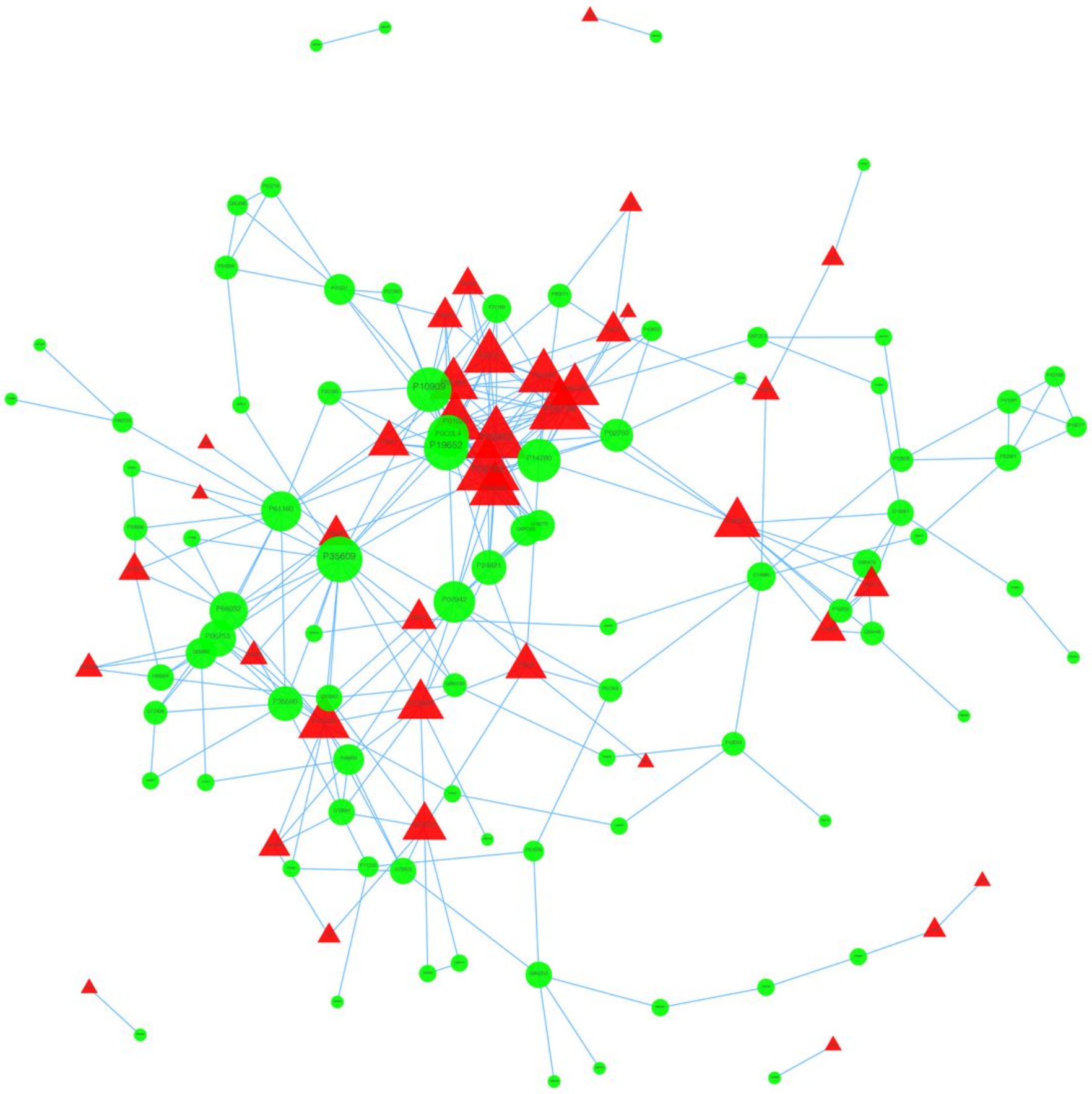

\section{Figure 5}

Visualization of PPIs between the SDAVF-DV and STV. Visualization of PPIs for the top 300 proteins using STRING analysis. Red represents upregulated proteins, and green represents downregulated proteins. 